\title{
GRAVIDEZ E SAÚDE MENTAL: UMA REVISÃO DE LITERATURA ACERCA DAS REPERCUSSÕES NA ADOLESCÊNCIA
}

\section{ARTIGO DE REVISÃO}

OLIVEIRA, Ketllen dos Santos ${ }^{1}$, SANTOS, Renato Maciel dos²

OLIVEIRA, Ketllen dos Santos. SANTOS, Renato Maciel dos. Gravidez e saúde mental: uma revisão de literatura acerca das repercussões na adolescência. Revista Científica Multidisciplinar Núcleo do Conhecimento. Ano. 06, Ed. 11, Vol. 06, pp. 05-16. Novembro 2021. ISSN: 2448-0959, Link de acesso: https://www.nucleodoconhecimento.com.br/psicologia/gravidez-e-saude, DOI: 10.32749/nucleodoconhecimento.com.br/psicologia/gravidez-e-saude

\section{RESUMO}

A maternidade na adolescência precisa ser observada como um processo históricosocial, não podendo ser considerada isoladamente. No Brasil, os dados estimam que entre mil adolescentes, quarenta e seis se tornaram mães. Nesse sentido, de que forma a gravidez repercute na saúde mental das adolescentes? Partindo dessa perspectiva, o presente estudo apresenta como objetivo geral: compreender através da literatura as repercussões da gravidez na saúde mental da adolescente. Para a realização da pesquisa, foi utilizado o método qualitativo, que além de descritivo, é fundamentalmente interpretativo. Tratou-se também de uma pesquisa bibliográfica, utilizando as plataformas Scielo e Biblioteca Digital Brasileira de Teses e Dissertações (BDTD), com os descritores: gravidez, adolescência e saúde mental. Após a busca, foram escolhidos trabalhos que tivessem o mesmo objetivo com a pesquisa, resultando em 4 materiais do Scielo e 9 da BDTD. A partir disso, observou-se que as representações sociais da gravidez na adolescência perpassam por questões como: cultura a respeito das funções maternas e papel de cuidadora;

\footnotetext{
${ }^{1}$ Pós-graduanda (em andamento) em Psicanálise, psicopatologia e saúde mental pelo Instituto Gaio. Graduação em Psicologia pelo Centro Universitário Estácio do Recife.

${ }^{2}$ Graduando em Psicologia pelo Centro Universitário Estácio do Recife.
}

RC: 100975

Disponível em: https://www.nucleodoconhecimento.com.br/psicologia/gravidez-esaude 
identificação entre mãe e filha; e a transgeracionalidade no que diz respeito à toda a estrutura familiar afetar o comportamento da adolescente, bem como as novas funções familiares desempenhadas. No que diz respeito às mudanças na vida da adolescente, foram identificadas mudanças sociais, corporais e sexuais, bem como a busca pela aquisição da identidade e abandono dos objetos infantis para entrada na vida adulta. Quanto às repercussões na saúde mental, a possibilidade de sintomas depressivos e ansiosos em adolescentes grávidas é duas vezes maior do que em adultas grávidas. Já entre as adolescentes que apresentaram ideação suicida, a maior parte eram solteiras e não contavam com apoio social. Concluiu-se que se torna imprescindível que as equipes de saúde possam identificar fatores de risco a fim de garantir o cuidado integral e promoção de saúde mental para esse grupo.

Palavras-chave: Gravidez, Adolescência, Saúde Mental.

\section{INTRODUÇÃO}

A adolescência é compreendida como um período de desenvolvimento e preparação para a vida adulta, já que ocorrem mudanças significativas no corpo, na vida social e emocional. Nesse sentido, a experimentação dos relacionamentos amorosos e do prazer sexual tendem a ocupar um espaço nessa fase da vida, sendo necessária uma maior atenção à ocorrência de gravidezes (VICENTIM, 2018). No Brasil, a gravidez na adolescência é um fenômeno que apresenta altos índices em comparação a outros países, estando 50\% acima da média mundial. Os dados estimam que entre mil adolescentes, quarenta e seis se tornaram mães (BRASIL, 2020).

Por ser uma etapa do desenvolvimento importante para a constituição da identidade, a adolescente gestante passa a lidar concomitantemente com a maternidade e a adolescência. Essa junção impõe novas responsabilidades e limitações, partindo da perspectiva em que a adolescência é um período exploratório de possibilidades antes da chegada da vida adulta. Além disso, também há mudanças no contexto 
familiar: além de filha, a jovem passa a ser mãe e a família também insere expectativas em relação ao exercício da sua maternidade (DIAS; TEIXEIRA, 2010).

É importante ressaltar que a gravidez na adolescência não é uma manifestação recente, já que ao longo do tempo grande parte das brasileiras vem tendo filhos nessa faixa e encerram seu ciclo reprodutivo de forma precoce através da laqueadura tubária (AQUINO et al., 2003). Ao fazer um recorte de classe, é possível perceber um prolongamento da juventude das classes mais favorecidas, uma vez que o tempo de estudo tende a ser maior em detrimento dos grupos populares, que, muitas vezes, precisam interromper os estudos e partir para o mercado de trabalho. Somada a isso, a desigualdade de gênero tende a socializar meninas para as atividades domésticas desde cedo, sendo a maternidade mais um reconhecimento desse papel social (AQUINO et al., 2003).

Em relação aos métodos contraceptivos, tem-se evidenciado que o não uso por adolescentes, na maioria das vezes, não decorre da falta de conhecimento ou falta de acesso. Fatores de ordem cognitiva como por exemplo: a crença que por ser adolescente não irá engravidar, ou fatores de ordem afetiva como desejos e motivações inconscientes podem influenciar na opção de não fazer uso de métodos anticoncepcionais. Muitas jovens também podem ter o receio de adotar medidas contraceptivas adequadas em suas primeiras relações sexuais, já que esse comportamento mostra uma busca ativa da mulher e esse é o oposto daquilo que é esperado socialmente (DIAS; TEIXEIRA, 2010).

A maternidade na adolescência, de acordo com Cafer (2016), precisa ser observada como um processo histórico-social, não podendo ser considerada isoladamente. Nesse sentido, por haver questões sociais, nem sempre a gravidez é indesejada ou problemática, pois pode estar ligada com a possibilidade de crescimento e elevação de status em seu grupo social, além do fato que cada adolescente possui particularidades e história de vida subjetiva. No entanto, a idade pode se apresentar como um fator de risco para o surgimento de depressão na gestação e no pós-parto,

RC: 100975

Disponível em: https://www.nucleodoconhecimento.com.br/psicologia/gravidez-esaude 
já que esse grupo tende a vivenciar eventos mais estressantes do que mães adultas (CAFER, 2016).

Partindo dessas considerações, a temática da gravidez na adolescência se apresenta de grande relevância para os pesquisadores, uma vez que envolve um processo complexo e multifatorial, sobretudo para a compreensão dos aspectos relacionados à saúde mental da adolescente que vivencia o processo de gestação, puerpério e maternidade. Nesse sentido, de que forma a gravidez repercute na saúde mental das adolescentes?

O presente artigo apresenta como objetivo geral: compreender os aspectos da gravidez na saúde mental da adolescente, bem como possui como objetivos específicos: a) descrever as representações sociais da gravidez na adolescência; b) apontar possíveis mudanças na vida da adolescente gestante; c) discorrer acerca dos impactos na saúde mental dessas adolescentes.

\section{MÉTODO}

Para a realização da pesquisa, foi destinado um método qualitativo, que além de descritivo, é fundamentalmente interpretativo, interagindo com os fenômenos sociais holisticamente (CRESWELL, 2010), de forma que não podem ser traduzidos em números e nem utilizados métodos estatísticos (ASSIS, 2009).

Tratou-se também de uma pesquisa bibliográfica, escolhida pois possibilita a quem pesquisa visualizar diversos fenômenos, sem limitar suas interpretações, para analisar e resolver o problema apresentado (GIL, 2019). Como afirmou Creswell (2010, p.46): "Ela fornece uma estrutura para estabelecer a importância do estudo e um indicador para comparar os resultados de um estudo com outros resultados". E, como base para essa comparação, é utilizado um levantamento bibliográfico, utilizando, como por exemplo: livros, artigos e periódicos (PIZZANI et al, 2012).

Para o levantamento bibliográfico desta pesquisa, foram escolhidas duas plataformas: a primeira é a principal plataforma digital de periódicos científicos da

RC: 100975

Disponível em: https://www.nucleodoconhecimento.com.br/psicologia/gravidez-esaude 
América Latina, a Scielo; e como segunda plataforma foi escolhida a Biblioteca Digital Brasileira de Teses e Dissertações, a BDTD. Em cada uma das plataformas, foi feita uma pesquisa com 03 descritores, que são: gravidez, adolescência e saúde mental.

$\mathrm{Na}$ Scielo foram encontrados 07 materiais; já na BDTD como resultado da pesquisa foram 11, entre teses e dissertações. Após essa primeira etapa, foi realizada uma leitura do resumo de todos os trabalhos científicos, para que fosse feita a exclusão daqueles que não condizem com os objetivos deste artigo. Como resultado final do levantamento de materiais, na Scielo restaram 4 artigos, enquanto no BDTD foram encontradas 02 teses e 07 dissertações selecionadas, que deram seguimento com a pesquisa.

\section{DESENVOLVIMENTO}

\subsection{REPRESENTAÇÕES SOCIAIS DA GRAVIDEZ NA ADOLESCÊNCIA}

De acordo com as representações sociais, cada pessoa é capaz de praticar um dinamismo social, não sendo apenas passivo socialmente, mas ativo para criar e mudar regras e papéis sociais (CAFER, 2016). Ainda segundo Cafer (2016), a cultura do indivíduo abrange sua moral, suas normas e seus conhecimentos, numa construção social-histórica, transmitida entre gerações de forma aprendida e compartilhada.

$\mathrm{Na}$ sua pesquisa, Cafer (2016) observou, nas jovens participantes, que seus discursos sobre o papel materno eram relacionados ao de cuidadora, de responsável pelos cuidados e alimentação do filho; trazendo através desses discursos a cultura a respeito das funções maternas. Apesar das falas observadas, no contexto investigado pela autora, a maioria das adolescentes não queriam vivenciar essa representação naquele momento.

RC: 100975

Disponível em: https://www.nucleodoconhecimento.com.br/psicologia/gravidez-esaude 
As mulheres de hoje vivem sob as representações de maternidade das mulheres do passado que viviam o modelo de mãe idealizada. Atualmente, as mulheres têm outros interesses e expectativas e outras formas para se realizarem como mulher e que não estão restritas à maternidade. Devido a essas representações idealizadas da maternidade, as mulheres contemporâneas entram em conflito com seu papel de mãe, o que acarreta sentimentos de dúvidas e angústias por não se atingir as expectativas de mãe perfeita (CAFER, 2016, p. 63).

Loss (2006), afirma que, na adolescência, as possibilidades do engravidamento pode-se estar relacionada a passagem nesses modos singulares de viver e ao início da atividade sexual. Também é afirmado que o processo da puberdade quebra o corpo infantil, ao mesmo tempo que adquire características do corpo da sua genitora, evidenciando a identificação entre mãe e filha. Esse processo de identificação vai além de características corporais, mas de papéis sociais e construções de vida semelhantes, assim como a transgeracionalidade da gravidez na adolescente (EWERTON, 2010).

Ewerton (2010), em seu material, vai além, afirmando que não apenas a mãe pode atuar no comportamento da adolescente, mas sim toda a estrutura familiar, incluindo todos os seus membros. Nos resultados da pesquisa da autora, o determinismo estrutural da interação da família interfere diretamente na escolha da gravidez.

No estudo com as gravidezes ocorrendo nas famílias de baixa renda, existe um movimento de mudança da estrutura familiar, em que a jovem mãe se torna também irmã mais velha do seu filho, criado pela avó, e assim sucessivamente nos papéis familiares (EWERTON, 2010). Essas mudanças dos papéis familiares acontecem de forma desordenada, gerando assim, uma dificuldade nas responsabilidades por parte da adolescente nos próximos estágios de vida, já que, os anteriores aconteceram de forma encurtada (EWERTON, 2010).

\subsection{MUDANÇAS NA VIDA DA ADOLESCENTE}

A gestação é um momento de transformações físicas, psicológicas, e sociais para a mulher (PICCININI et al., 2008). São meses de preparo e muitas vezes isso acarreta

RC: 100975

Disponível em: https://www.nucleodoconhecimento.com.br/psicologia/gravidez-esaude 
algumas mudanças no estilo de vida, a fim de preservar a saúde gestacional. Segundo Coutinho (2014), muitas deixam de consumir álcool e outras drogas, adotam uma dieta mais saudável, expõem-se menos a perigos e se esforçam ao máximo para preservar a própria saúde, e a do bebê.

Em um estudo realizado por Santos (2014), sobre a qualidade de vida em adolescentes gestantes, alguns domínios foram abordados: domínio físico (dor, desconforto, repouso), psicológico (autoestima, processos psicológicos básicos, sentimentos, espiritualidade), relações sociais (atividade sexual, suporte social) e meio ambiente (segurança, lazer, recursos financeiros, transporte). A autora apontou que gestantes adolescentes do primeiro filho apresentaram escores médios significativamente menores em todos os domínios apontados, em comparação a adolescentes com histórico de gestações anteriores; evidenciando que a primeira gestação é um evento que repercute diretamente na percepção da qualidade de vida das adolescentes.

Um fato comum da gravidez na adolescência é a união conjugal forçada pelos familiares dos adolescentes, já que na maioria das vezes a gestação ocorre quando a adolescente ainda reside com sua família de origem, gerando mudanças na estrutura familiar (BORGES, 2007). A autora refere-se ainda aos hábitos das adolescentes que costumavam ir a festas e estar com os amigos, mas que agora passam a ter novas responsabilidades (BORGES, 2007). Outras consequências sociais da gravidez nessa fase da vida podem se dar na dificuldade para acessar o mercado de trabalho qualificado e para retornar à escola (FREITAS, 2007)

A entrada na adolescência é perpassada pela mudança corporal, que passa a ter crescimento das mamas, surgimento dos pêlos, arredondamento dos quadris. Com a gestação, novas mudanças corporais aparecem e a adolescente passa a se perceber com um novo corpo grávido, que muitas vezes pode gerar desconforto e/ ou sinal de poder e autoconfiança em alguns casos. Uma preocupação que também tende a ficar em evidência é com o corpo no período do pós-parto (BORGES, 2007). 
Atreladas às mudanças corporais, muitas vezes a adolescente pode experimentar uma diminuição do desejo sexual e de um maior desconforto com a barriga ou receio de causar danos ao bebê. Em alguns casos o parceiro não entende essas mudanças e a mulher tende a ceder às insistências para manter relações sexuais (BORGES, 2007).

A busca pela aquisição da identidade e abandono dos objetos infantis, pode levar os adolescentes ao desejo de pular essa fase da vida e iniciar as atividades e responsabilidades dos adultos (FREITAS, 2001). Desse modo, a experiência da gravidez pode ser entendida como uma entrada para o mundo adulto e traz consigo essa série de mudanças que podem repercutir diretamente na saúde mental das adolescentes grávidas.

\subsection{REPERCUSSÕES NA SAÚDE MENTAL DAS ADOLESCENTES}

A saúde mental das gestantes por vezes é um assunto ignorado por dois fatores principais: o primeiro se refere ao pensamento do senso comum de que a gravidez é um período de bem-estar e realização, acreditando que a gravidez poderia ser um fator protetivo da saúde mental dessas mulheres; o segundo se destina ao enfoque nos transtornos psiquiátricos no período do pós-parto. No entanto, transtornos psiquiátricos na gestação e no pós-parto possuem prevalência semelhante (SANTOS, 2014)

Ainda de acordo com Santos (2014), a possibilidade de sintomas depressivos e ansiosos em adolescentes grávidas é duas vezes maior do que em adultas grávidas. Esses dados podem estar relacionados à puerilidade e às mudanças advindas da gestação, como a necessidade de afastamento da escola e o abandono dos estudos em alguns casos.

Em um estudo comparativo sobre problemas de saúde mental entre jovens grávidas e não-grávidas, Caputo e Bordin (2007) estimaram que adolescentes grávidas e em sua primeira gestação apresentaram com mais frequência sintomas de ansiedade,

RC: 100975

Disponível em: https://www.nucleodoconhecimento.com.br/psicologia/gravidez-esaude 
depressão e constituíam maior número de fumantes em relação a adolescentes sexualmente ativas e que não estavam grávidas.

Um estudo realizado por Freitas e Botega (2002), com adolescentes dos três trimestres gestacionais, apontou que quase metade das jovens que se sentiam felizes e realizadas com a gravidez, apresentaram o desejo de engravidar e mantinham relações estáveis com seu companheiro. Já entre as adolescentes que apresentaram ideação suicida, a maior parte eram solteiras e não contavam com apoio social. Nesse sentido, a rede de apoio pode ser considerada um fator protetivo para as jovens.

Quanto aos dados relacionados à ansiedade nas adolescentes do estudo, nem todas apresentaram sintomas ansiosos e não foram observadas mudanças significativas entre os três trimestres de gestação. No que se refere à depressão, a falta de apoio e o sentimento de não serem importantes para alguém se mostrou presente nas jovens. Um ponto importante para ser analisado dentre algumas das adolescentes com sintomas depressivos, é ver a possibilidade de acabar com a solidão tendo um filho, ou seja, gerar sua própria família e rede de apoio (FREITAS; BOTEGA, 2002).

Um outro estudo realizado por Caputo e Bordin (2008), analisou a relação entre a gravidez na adolescência e o uso frequente de álcool e drogas no contexto familiar. Foi apontado que o uso abusivo dessas substâncias na família pode funcionar como um fator estressante, além de apresentar como consequências uma maior frequência de agressões físicas e brigas entre os pais, evidenciando assim, que a maioria das adolescentes grávidas nesse contexto não possuíam a família como ponto de informações e apoio à questões relacionadas à sexualidade e métodos preventivos.

Para adolescentes que vivem em abrigos ou ambientes caóticos, as condições financeiras geralmente são precárias, muitas vezes não contam com apoio e podem ser frequentemente expostas a abusos sexuais. Todos esses fatores podem gerar o 
sentimento de insegurança e ambivalência, resultando em estratégias de enfrentamento e fuga no uso de álcool/drogas e no comportamento sexual de risco, aumentando as chances de gravidez na adolescência dessa população (SCAPPATICCI; BLAY, 2010).

Freitas (2007) em um estudo a respeito do comportamento suicida em adolescentes grávidas aponta que a ideação suicida é mais frequente em adolescentes com grande sofrimento psicossocial, estando diretamente ligado com a desesperança, ansiedade e histórico de tentativas de suicídio. Também evidenciou que adolescentes que perderam um dos pais na infância compõem um grupo de risco ao comportamento suicida.

É importante ressaltar que o estado emocional da gestante, quando associados aos fatores ambientais podem acarretar uma série de complicações, tais como: parto prematuro, prejuízos na relação mãe-bebê, prejuízos no manejo com o filho e consequências na saúde mental da criança (TRETTIM, 2019).

\section{CONSIDERAÇÕES FINAIS}

Este estudo possibilitou um maior entendimento acerca da temática da gravidez na adolescência, e seus impactos na saúde mental das mesmas. A adolescência em si, já é considerada como um período de mudanças, amadurecimento mental e físico, em que o indivíduo busca representatividade para entrar na idade adulta.

Na pesquisa, foi observado que o Brasil possui uma elevada taxa de gravidez na adolescência, sendo constatado como um fenômeno que envolve desde um problema de classe, até uma troca de papéis dentro da própria família. Também se observou que as representações sociais da gravidez na adolescência perpassam por questões como: cultura a respeito das funções maternas e papel de cuidadora; identificação com as características de sua genitora; e a transgeracionalidade no que diz respeito à toda a estrutura familiar afetar o comportamento da adolescente, bem como as novas funções familiares desempenhadas. 
Entre os resultados, destacaram-se que as jovens que contavam com uma rede de apoio efetiva, se sentiam mais realizadas com a maternidade. Já aquelas que não possuíam esse apoio social, possuem maiores chances de apresentarem sintomas de ansiedade, depressão, ideação suicida e sentimento de desesperança. Nesse sentido, torna-se imprescindível que as equipes de saúde possam identificar fatores de risco durante a realização do pré-natal de adolescentes grávidas, a fim de garantir o cuidado integral e promoção de saúde mental para esse grupo.

\section{REFERÊNCIAS}

AQUINO, Estela et al. Adolescência e reprodução no Brasil: a heterogeneidade dos perfis sociais. Cad. Saúde Pública, Rio de Janeiro: v. 19, n. 2, p. 377-388, 2003. Disponível em: https://www.scielosp.org/article/csp/2003.v19suppl2/S377S388/pt/\#ModalArticles. Acesso em: 10/04/2021.

ASSIS, Maria Cristina. Metodologia do trabalho científico. São Paulo, 2009. 48 p.

BORGES, Juliana de Moura. Coping e saúde mental de grávidas adolescentes participantes do projeto meninas de luz. Dissertação (Mestrado) - Curso de Psicologia, PUC Goiás, Goiás: 144 f. 2007. Disponível em: https://bdtd.ibict.br/vufind/Record/PUC_GO_1830d89bb6e8b41c39397994ea45b9e9. Acesso em: 25/05/2021.

BRASIL. Campanha visa reduzir altos índices de gravidez precoce no Brasil. 2020. Disponível em: https://www.gov.br/mdh/pt-br/assuntos/noticias/20202/fevereiro/campanha-visa-reduzir-altos-indices-de-gravidez-precoce-no-brasil. Acesso em: 29 mar. 2021.

CAFER, Juliana Regina. Representações sociais sobre amamentação na perspectiva de mães adolescentes com sintomas de depressão pós-parto. Dissertação (Mestrado) - Curso de Enfermagem, USP, Ribeirão Preto: 86 f., 2016. Disponível em: https://teses.usp.br/teses/disponiveis/22/22133/tde-30092016155210/pt-br.php. Acesso em: 13/06/2021.

RC: 100975

Disponível em: https://www.nucleodoconhecimento.com.br/psicologia/gravidez-esaude 
CAPUTO, Valéria Garcia; BORDIN, Isabel Altenfelder. Problemas de saúde mental entre jovens grávidas e não- grávidas. Rev Saúde Pública, Marília: v. 41, n. 4, p. 573-581, 2007. Disponível em: https://www.scielo.br/j/rsp/a/hBWjFNYFDhX4s5Fg9rBnGWm/abstract/?lang=pt\#: :te $\mathrm{xt}=$ Comparado\%20\%C3\%A0s\%20adolescentes\%20n\%C3\%A30\%2Dgr\%C3\%A1vid as,0\%25\%3B\%20p\%3D0\%2C002). Acesso em: 05/05/2021.

CAPUTO, Valéria Garcia; BORDIN, Isabel Altenfelder. Gravidez na adolescência e uso freqüente de álcool e drogas no contexto familiar. Rev Saúde Pública, Marília: v. $42, \quad$ n. $3, \quad$ p. $402-410,2008$. Disponível em: https://www.scielo.br/j/rsp/a/fBDXm8XMQbgKM5xKwZpQfJP/abstract/?lang=pt\#: :te $\mathrm{xt}=$ CONCLUS\%C3\%95ES\%3A\%200\%20uso\%20freq\%C3\%BCente\%20de,presen\% C3\%A7a\%20de\%20baixa\%20escolaridade\%20materna. Acesso em: 10/04/2021.

COUTINHO, Emília de Carvalho, et al. Mudanças no estilo de vida provocadas pela gravidez e parto. Atas - Investigação qualitativa em Saúde, Portugal: v.2, p.382387, $2014 . \quad$ Disponível em: https://proceedings.ciaiq.org/index.php/CIAIQ/article/view/553/548 Acesso em: 15/07/2021.

CRESWELL, John. Projeto de pesquisa: métodos qualitativo, quantitativo e misto; tradução Magda Lopes. º edição. Porto alegre: Artmed, 2010, 296 páginas.

DIAS, Ana Cristina Garcia; TEIXEIRA, Marco Antônio Pereira. Gravidez na adolescência: um olhar sobre um fenômeno complexo. Paidéia, Riberão Preto: v. 20, n. 45, p. 123-131, 2010. Disponível em: https://doi.org/10.1590/S0103863X2010000100015. Acesso em: 27/03/2021.

EWERTON, Mary Lúcia Adler. Estrutura familiar e gravidez na adolescência: um enfoque sistêmico. Dissertação (Mestrado) - Curso de Ciências Biológicas, UFMA, São Luiz: 114 f., 2010. Disponível em: https://tedebc.ufma.br/jspui/handle/tede/1147. Acesso em: 25/05/2021. 
FREITAS, Gisleine Vaz Scavacini. Prevalencia de ansiedade, depressão e ideação suicida numa população de adolescentes gravidas. Dissertação (Mestrado) - Curso de Ciências Biomédicas, UNICAMP, Campinas: 110 f., 2001. Disponível em: http://repositorio.unicamp.br/handle/REPOSIP/311451. Acesso em: $28 / 07 / 2021$

FREITAS, Gisleine Vaz Scavacini. Comportamento suicida em adolescentes grávidas: um estudo de caso-controle. Tese (Doutorado) - Curso de Ciências Biomédicas, UNICAMP, Campinas:184 f, 2007. Disponível em: http://repositorio.unicamp.br/jspui/handle/REPOSIP/311454. Acesso em: 25/05/2021.

FREITAS, Gisleine Vaz Scavacini; BOTEGA, Neury José. Gravidez na adolescência: prevalência de depressão, ansiedade e ideação suicida. Rev Assoc Med Bras, Campinas: v. $48, \quad$ n. 3 , p. 245-249, 2002. Disponível em: https://www.scielo.br/j/ramb/a/KLLN46j6JSRnX7hR7YQbnPg/?lang=pt Acesso em: 09/04/2021

GIL, Antônio Carlos. Como Elaborar Projetos de Pesquisas. 6. ed. São Paulo: Atlas, 2019. $173 \mathrm{p}$.

LOSS, Maria Aparecida. As possibilidades do engravidamento na adolescência: um desafio à integralidade nas práticas em saúde pública. Dissertação (Mestrado) Curso de Psicologia, UFRGS, Porto Alegre: 198 f, 2006. Disponível em: https://lume.ufrgs.br/handle/10183/7453?show=full. Acesso em: 15/04/2021.

PICCININI, Cesar Augusto et al. Gestação e a constituição da maternidade. Psicologia em Estudo. Maringá: v.13, n.1, p.63-72, 2008. Disponível em: https://doi.org/10.1590/S1413-73722008000100008. Acesso em: 12/04/2021.

PIZZANI, Luciana. et al. A arte da pesquisa bibliográfica na busca do conhecimento. Revista Digital de Biblioteconomia e Ciência da Informação, [s.l.]: v. 10, n. 1, 53p, 10 jul. 2012. Universidade Estadual de Campinas. Disponível em: 
https://periodicos.sbu.unicamp.br/ojs/index.php/rdbci/article/view/1896 Acesso em: 09/04/2021.

SANTOS, Marina dos. Qualidade de vida em gestantes adolecentes: correlatos sociodemográficos, obstétricos e psiquiátricos. Dissertação (Mestrado) - Curso de Departamento de Saúde e Comportamento, UCPEL, Pelotas: 75 f., 2014. Disponível em: https://bdtd.ibict.br/vufind/Record/UCPe_34b67f2364b6f9fe687e50cc060f97f6. Acesso em: 21/07/2021.

SCAPPATICCI, Anne Lise Sandoval Silveira; BLAY, Sergio Luis. Mães adolescentes em situação de rua: uma revisão sistemática da literatura. Rev Psiquiatr Rs, São Paulo: v. $32, \quad$ n. 1 , p. 3-15, 2010. Disponível em: https://www.scielo.br/j/rprs/a/RrtB3sFH7NMzsXYyxWpX5Nn/abstract/?lang=pt Acesso em: 05/05/2021.

TRETTIM, Jéssica Puchalski. Suicidalidade e depressão materna: impacto no desenvolvimento infantil. Tese (Doutorado) - Curso de Departamento de Saúde e Comportamento, UCPEL, Pelotas: 104 f. 2019. Disponível em: http://bdtd.ibict.br/vufind/Record/UCPe_31ab48b86231927f55c6be038aba631f. Acesso em: 14/04/2021.

VICENTIM, Alessandra Lima. Aspectos Associados à Gravidez na Adolescência. Dissertação (Mestrado) - Curso de Enfermagem, FAMERP, São José do Rio Preto: 95 f, 2018. Disponível em: https://bdtd.famerp.br/handle/tede/563. Acesso em: 23 jun. 2021.

Enviado: Setembro, 2021.

Aprovado: Novembro, 2021.

RC: 100975

Disponível em: https://www.nucleodoconhecimento.com.br/psicologia/gravidez-esaude 